\title{
Autonomous Rendezvous and Docking of Two 3U Cubesats Using a Novel Permanent-Magnet Docking Mechanism
}

\author{
Jing Pei, Luke Murchison†, Adam BenShabat: Victor Stewart\$, \\ James Rosenthal, Mark Banchy, and Drew Sellers** \\ NASA Langley Research Center, Hampton, Virginia, 23681 \\ Ryan Elandt, David Sawyer Elliott $\dagger^{\dagger}$ \\ Mechanical and Aerospace Engineering Department, Cornell University, Ithaca, New York, 14853 \\ Adam Weber $\ddagger$
Department of Atmospheric, Oceanic and Space Sciences, University of Michigan, Ann Arbor, Michigan, 48109
}

Small spacecraft autonomous rendezvous and docking is an essential technology for future space structure assembly missions. A novel magnetic capture and latching mechanism is analyzed that allows for docking of two CubeSats without precise sensors and actuators. The proposed magnetic docking hardware not only provides the means to latch the CubeSats but it also significantly increases the likelihood of successful docking in the presence of relative attitude and position errors. The simplicity of the design allows it to be implemented on many CubeSat rendezvous missions. Preliminary Monte Carlo simulation shows that the proposed docking design leads to successful docking under 3-sigma dispersions for all key system parameters. On the other hand, successful docking is not possible without the aid of the proposed docking subsystem. Future experiments are to be performed at the 3 Degrees of Freedom flat-floor facility at NASA Langley Research Center to demonstrate this novel concept. Extensive simulation and ground testing will provide sufficient confidence that the proposed docking mechanism will perform as expected in the space environment.

\section{Introduction}

In the aerospace industry, there has been increasing interest in low-budget space missions involving CubeSats. ${ }^{1-5}$ Assembly of multiple CubeSats in space into large structures such as a space telescope or solar panels could be tremendously beneficial for the science and engineering communities. Several research groups $^{6-9}$ are currently tackling the challenges associated with autonomous rendezvous and docking of small scaled spacecraft on orbit. To the best of the authors' knowledge, the U.S. has never successfully performed an autonomous rendezvous and docking procedure. ${ }^{10,11}$ The On-orbit Autonomous Assembly of Nanosatellites (OAAN) team at NASA Langley was formed in response to the Announcement of Opportunity for the pilot Early Career Initiative from the Space Technology Mission Directorate (STMD) at NASA Headquarters. The

\footnotetext{
*Aerospace Engineer, Systems Analysis and Concepts Directorate, Vehicle Analysis Branch

${ }^{\dagger}$ Aerospace Engineer, Engineering Directorate, Structural and Thermal Systems Branch

¥Aerospace Engineer, Engineering Directorate, Structural and Thermal Systems Branch

$\S$ Aerospace Engineer, Engineering Directorate, Mechanical System Branch

๙ Aerospace Engineer, Engineering Directorate, Electronic Systems Branch

$\|$ Aerospace Engineer, Engineering Directorate, Flight Software Systems Branch

**Aerospace Engineer, Engineering Directorate, Flight Software Systems Branch

${ }^{\dagger} \mathrm{PhD}$ Candidate, Cornell University

$\ddagger \ddagger$ MEng Candidate, University of Michigan
} 
idea is to use permanent magnets to perform the alignment and locking of two CubeSats during the final phase of a docking maneuver will make the docking process robust in the presence of system uncertainties.

At this time, the state of the art offers no proven systems for docking small-scaled spacecrafts. Given the size and power constraints on a $3 \mathrm{U}$ CubeSat, permanent magnets were chosen over electromagnets because they require no power to operate and reduce system complexity. For relative navigation, the OAAN system utilizes Carrier-Phase Differential Global Position System (CDGPS) ${ }^{12}$ to determine the relative position of the two CubeSats by measuring the phase difference of the GPS carrier wave at two locations, which provides relative position measurement within 2-centimeter accuracy. Currently most rendezvous and docking approaches require operators in the loop or visual navigation methods. CDGPS makes OAAN tolerant to ground communication interruptions, network failures, and latencies associated with human-inthe-loop control systems. It is superior to visual navigation methods in terms of cost, complexity, size, mass, and power requirements. Figure 1 shows the standard $3 \mathrm{U}+$ CubeSat configuration. The cylindrical can on the $-Z$ face of the spacecraft is the proposed docking subsystem.

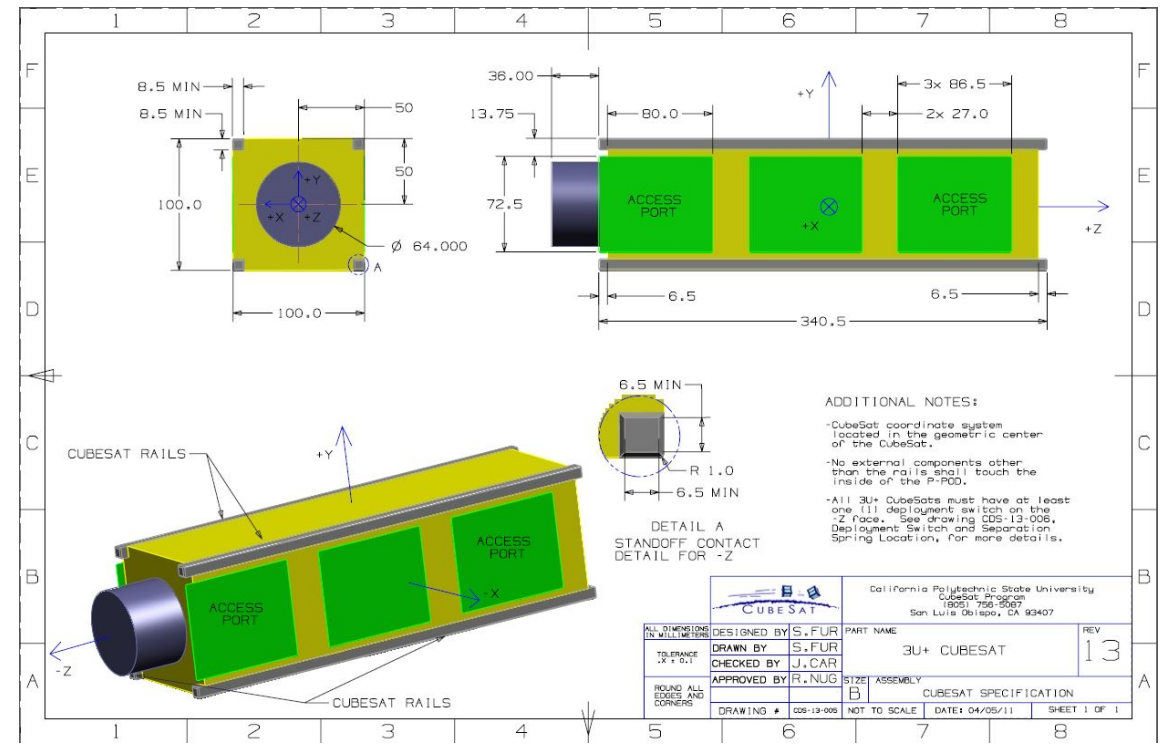

Figure 1. 3U+ CubeSat Schematic

Figure 2 shows the OAAN concept of operation and the adopted axis system during the final docking phase. Both the leader and follower CubeSats will be aligned with the $Z$ body axis directed along the local v-bar direction of the LVLH (Local Vertical Local Horizontal) frame. The translational controller will bring the follower towards the leader at $\mathrm{O}$ (on the order of) $\mathrm{cm} / \mathrm{s}$. Once the CubeSats are within the basin of attraction of the magnetic docking subsystem, where magnetic forces and moments dominate the dynamics, feedback control will be turned off allowing for passive elimination of residual relative position and attitude errors. The CubeSats will then align and complete the docking process. The following terminology will be used to describe the relative separation between the CubeSats throughout this paper: $Z$ describes the relative axial separation, $X$ describes the lateral separation which is aligned with the r-bar direction pointing towards the center of the earth, and $Y$ completes the right handed triad. 


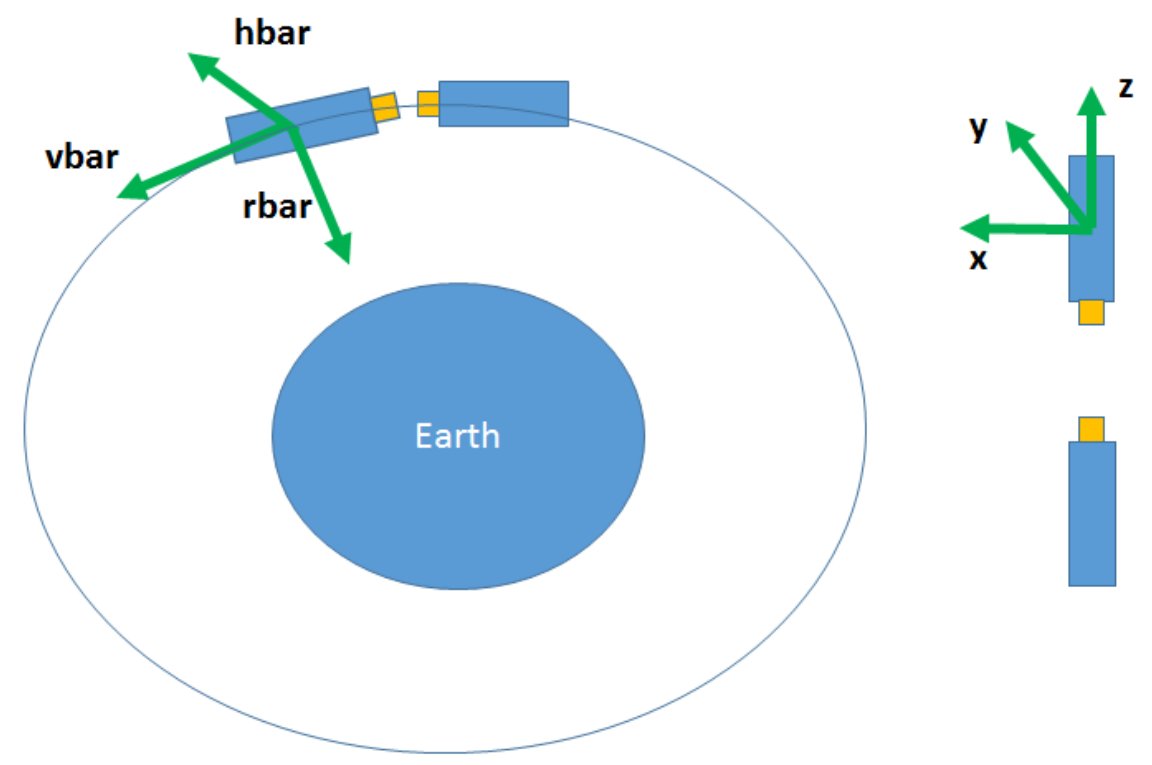

Figure 2. Docking Concept of Operation

The remainder of this paper is organized as follows: Section II describes key system parameters and uncertainties that successful docking is sensitive towards. Trade studies were performed to obtain the current magnetic docking design that maximizes the basin of attraction while minimizing effects on other subsystems. Section III describes the modeling of the magnetic docking subsystem and the capture volume. Section IV presents preliminary simulation results of the final docking phase using the Clohessy-Wiltshire equations and describe an energy based approach used to analyze the docking process. Section V summarizes the results and provides discussion of ongoing work.

\section{Key Dynamics Models}

\section{A. Relative position navigation}

Due to communication constraints, only relative position measurements are available between the leader and follower CubeSat. CDGPS allows for $\mathrm{O}(\mathrm{cm})$ relative positioning accuracy. The Swift Navigation Piksi ${ }^{12}$ is the commercial CDGPS hardware solution that was selected for OAAN. The Piksi, more than any other component, is paramount to the success of the OAAN mission. There are few alternatives to CDGPS technology (visual methods are out of the scope of the project). The Piksi units require to have one external antenna that faces away from the Earth to point toward the GPS satellites. It outputs only the relative position between the two phase centers of the Piksi GPS antenna.

Data produced by the Piksi includes the following errors: 1) Random noise. This error is not driven by a stochastic process and changes for each measurement. In the navigation software the noise is smoothed with a Kalman Filter. 2) Bias error from GPS satellite constellation geometry. Docking success is most sensitive to this stochastic error and tests indicate that this error is $\mathrm{O}(\mathrm{cm})$. 3) Error from incorrect Integer Ambiguity Problem(IAP) solution. The third error can range from a few centimeters to tens of meters in relative position. It can occur both suddenly during operation or arise during initialization of the Piksi hardware. Due to its rare occurrence, it is not considered part of the nominal docking scenario. However, ongoing work is being performed to mitigate impact of this error type occurring during operations..

\section{B. Attitude and Position Control}

The Attitude Determination and Control System (ADCS) is provided by Blue Canyon Technologies (BCT). The $\mathrm{XACT}^{13}$ unit is a commercial-off-the-shelf product with flight heritage. The XACT provides precise three-axis attitude control using reaction wheels, magnetic torque rods, and integrated control algorithms. Figure 3 is an overview of the XACT ADCS unit. During operation, the attitude knowledge degrades at 
roughly 3 degrees per hour if the star tracker is not operational (i.e. an occluded field of view)..

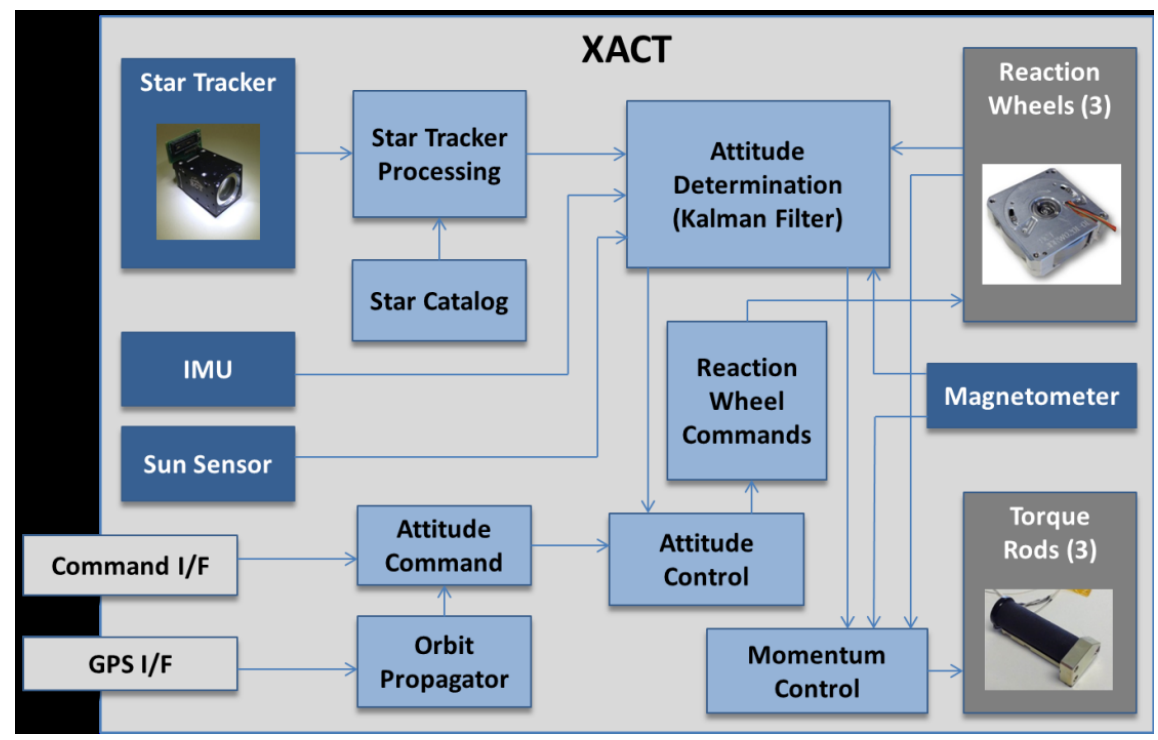

Figure 3. ADCS overview ${ }^{13}$

For translational control, a four cold gas thruster layout design is used such that the thrusters provide pure translational motion. The thrusters are strategically placed at the corners of an imaginary tetrahedron with the thrust vector directed towards the center of mass. A simple control allocation and pulse width modulation scheme is used to convert the commanded force into thruster ON time. Disturbance torques due to thrust vector misalignment and other hardware imperfections will be accounted for by the ADCS. Thruster minimum impulse bit (MIB) is a major factor in determining the precision and accuracy that the translational control loop could achieve. ${ }^{14}$ A $30 \mathrm{~ms}$ MIB is assumed in the current simulations.

\section{Magnetic Docking Subsystem}

It is intuitive from simplicity and a pure docking success standpoint to use a single large permanent magnet per docking subsystem. Doing so would make the system most robust to residual relative errors in the attitude and position loops. Practical constraints such as volume, mass, and the impact a strong electromagnetic field on other subsystems provides a hard limit on the size of the magnet. From an attitude control perspective, a single magnetic dipole on board the CubeSat is problematic because a disturbance torque will be generated due to its interaction with the magnetic field of the Earth shown in Eq.(1) ${ }^{14}$

$$
\tau_{d}=M \times B
$$

where $M$ is the net magnetic dipole moment of the docking subsystem and $B$ is the Earth's magnetic field. A 0.5 inch cuboid magnet would generate a disturbance torque that is greater than the available control torque from both the reaction wheels and the magnetic torque rods. Due to this constraint, the docking subsystem is required to have a nominally zero net dipole moment. The next best choice is to have a pair of magnets per docking subsystem with the dipoles having opposite directions in the $Z$ axis of the body frame. Figure 4 shows a schematic of the dock system. The capture range associated with this design is smaller compared to the single dipole design due to the existence of unstable equilibrium conditions in which the two docking subsystems would repel each other. 


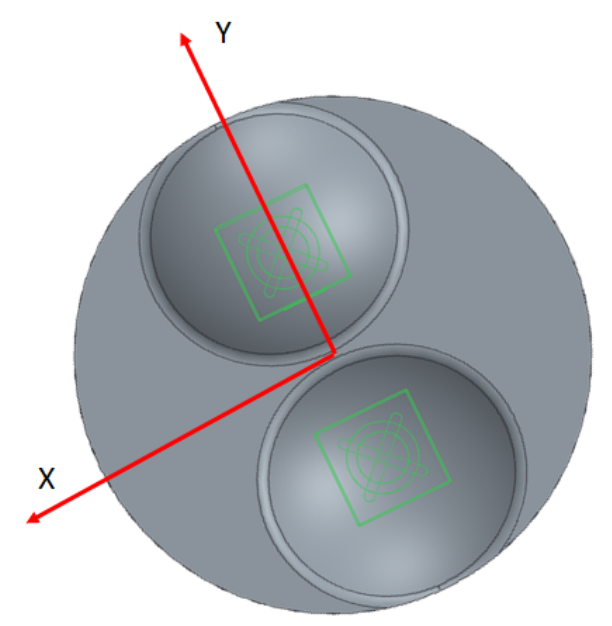

Figure 4. Magnetic Docking Subsystem Schematic

In addition to the zero net magnetic dipole requirement, the ADCS also requires that the magnetic docking subsystem shall not generate a changing magnetic field at the location of the magnetometer that is significant when compared to the geomagnetic field vector of the earth. For one degree of pointing accuracy, the magnetic field induced at the magnetometer location should be less than $0.7 \mu \mathrm{T}$. This further provides an upper bound on the strength of the magnetic dipoles. Equation.( 2$)$ is used to estimate the magnetic field $(B)$ induced at a certain distance $(\hat{r})$ by a magnetic dipole moment of strength $(\vec{m})$.

$$
B=\frac{\mu_{o}}{4 \pi}\left[3 \frac{(\vec{m} \cdot \hat{r}) \hat{r}-\vec{m}}{|r|^{3}}\right]
$$

\section{Magnetic docking subsystem model}

The capture volume needs to be quantified to understand the robustness of the magnetic docking subsystem. The capture volume is defined as the set of initial conditions (in terms of relative position, velocity, attitude, and attitude rates) for which the docking subsystem will cause the CubeSats to dock successfully without the aid of feedback control. In order to simplify this 12 dimensional initial condition problem, it can be reasonably assumed that the relative attitude errors and rates have been nulled out by the ADCS and position control is able eliminate any excessive radial velocity. Doing so simplifies the capture volume down to 4 variables: relative position and relative axial velocity.

References ${ }^{15,16}$ provide analytical expressions for the force and moment produced by two magnetic dipoles and are provided in Eq.( 3) and Eq.( 4), respectively. These expressions are used to approximate the capture volume between the docking subsystems and in the GNC simulations. Here $\hat{r}$ is the unit position vector from dipole a to dipole $\mathrm{b}$ and $\hat{m}$ is the magnetic dipole moment unit vector. It is important to note here that $\tau_{a b}$ is NOT equal to $-\tau_{b a}$.

$$
\begin{gathered}
\vec{F}=\frac{3 \mu_{o} m_{a} m_{b}}{4 \pi|\vec{r}|^{4}}\left(\hat{r}\left(\hat{m}_{a} \cdot \hat{m}_{b}\right)+\hat{m}_{a}\left(\hat{r} \cdot \hat{m}_{b}\right)+\hat{m}_{b}\left(\hat{r} \cdot \hat{m}_{a}\right)-5 \hat{r}\left(\hat{r} \cdot \hat{m}_{a}\right)\left(\hat{r} \cdot \hat{m}_{b}\right)\right) \\
\vec{\tau}_{a b}=\frac{\mu_{o} m_{a} m_{b}}{4 \pi|\vec{r}|^{3}}\left(3\left(\hat{m}_{a} \cdot \hat{r}\right)\left(\hat{m}_{b} \times \hat{r}\right)+\left(\hat{m}_{a} \times \hat{m}_{b}\right)\right)
\end{gathered}
$$

Equation.( 3) suggests that for two magnetic dipoles pointing in the same direction, the capture volume can be graphically approximated by a cone as shown in Fig. 5. Generally speaking, if the relative position of the two CubeSats falls within this cone of influence, the magnetic forces would cause the spacecraft to attract in all 3 axes. Once outside the cone, there exist 2 regions (dependent upon the dot product between $\hat{r}, \hat{m}_{a}$, and $\hat{m}_{b}$ ). Region I: repulsion in $X$ with attraction in $Y$ and $Z$. Region II: repulsion in all 3 axes. A energy-based approach describe in Section IV provides a deep analytical view of the docking subsystem. 


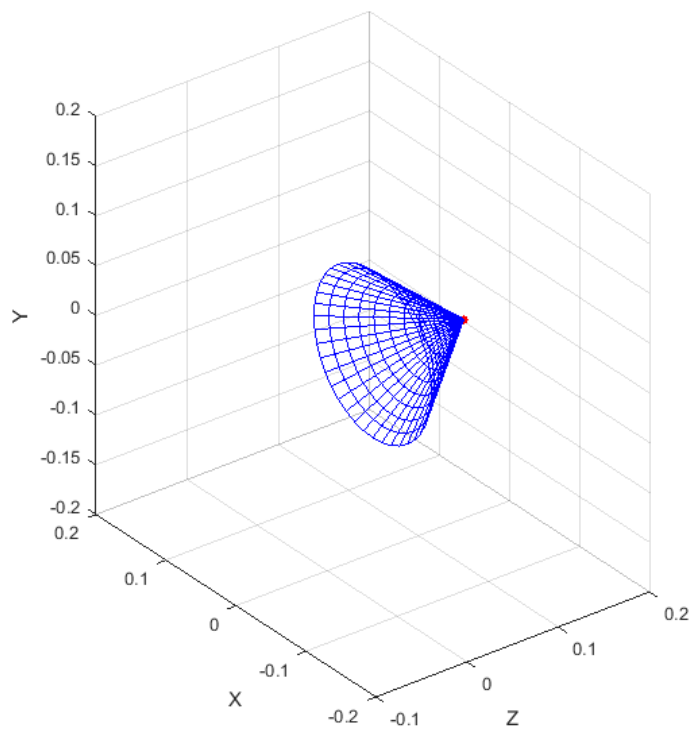

Figure 5. Analytical Capture Volume: 1 Cuboid Magnet Design

With a pair of cuboid magnets on each CubeSat, the capture volume becomes more complicated. Using the same analogy as above, the analytic capture volume can be approximated by the union of two cones (one centered at the location of each dipole), as shown in Fig. 6. It is important to note that, though the basin of attraction stretches out towards infinity, the attractive force varies with $r^{4}$ according to Eq. (3). In reality the effective length of the cone is contingent upon the strength and size of the magnets.

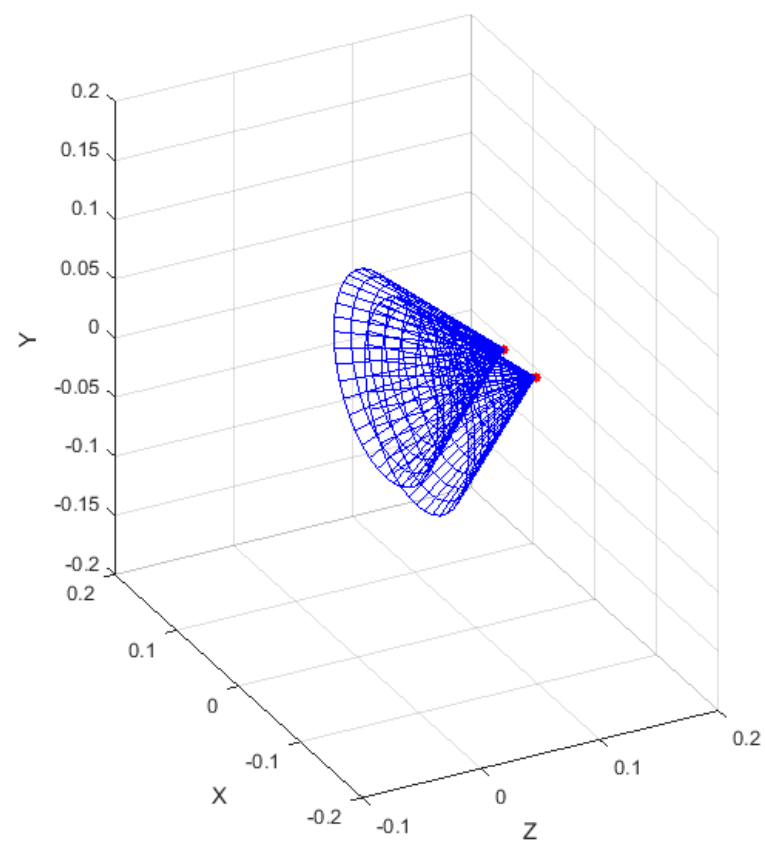

Figure 6. Analytical Capture Volume: 2 Cuboid Magnet Design 
In order to get a time domain understanding of the capture volume, an open-loop 12 DOF simulation was developed that includes the CubeSat mass properties and models of the magnetic forces and moments using the dipole equations. The simulation was initialized with the two CubeSats separated by less than $30 \mathrm{~cm}$ in $\mathrm{Z}$ with some relative radial offset $(<20 \mathrm{~cm})$ and relative velocity in the $\mathrm{Z}$ direction. Docking was considered successful if the centerlines of the docking hardwares come within $1 \mathrm{~cm}$ of each other in the axial (Z) direction and with maximum radial offset of $1 \mathrm{~cm}$. Figure. 7 shows the simulated capture volume (shown in blue) with an initial axial velocity of $1 \mathrm{~cm} / \mathrm{s}$. The conical shape (with the vertex located at the origin) agrees well with the analytical two dipole model. The cone angle is dependent on the separation distance between the top and bottom magnets in the docking subsystem.

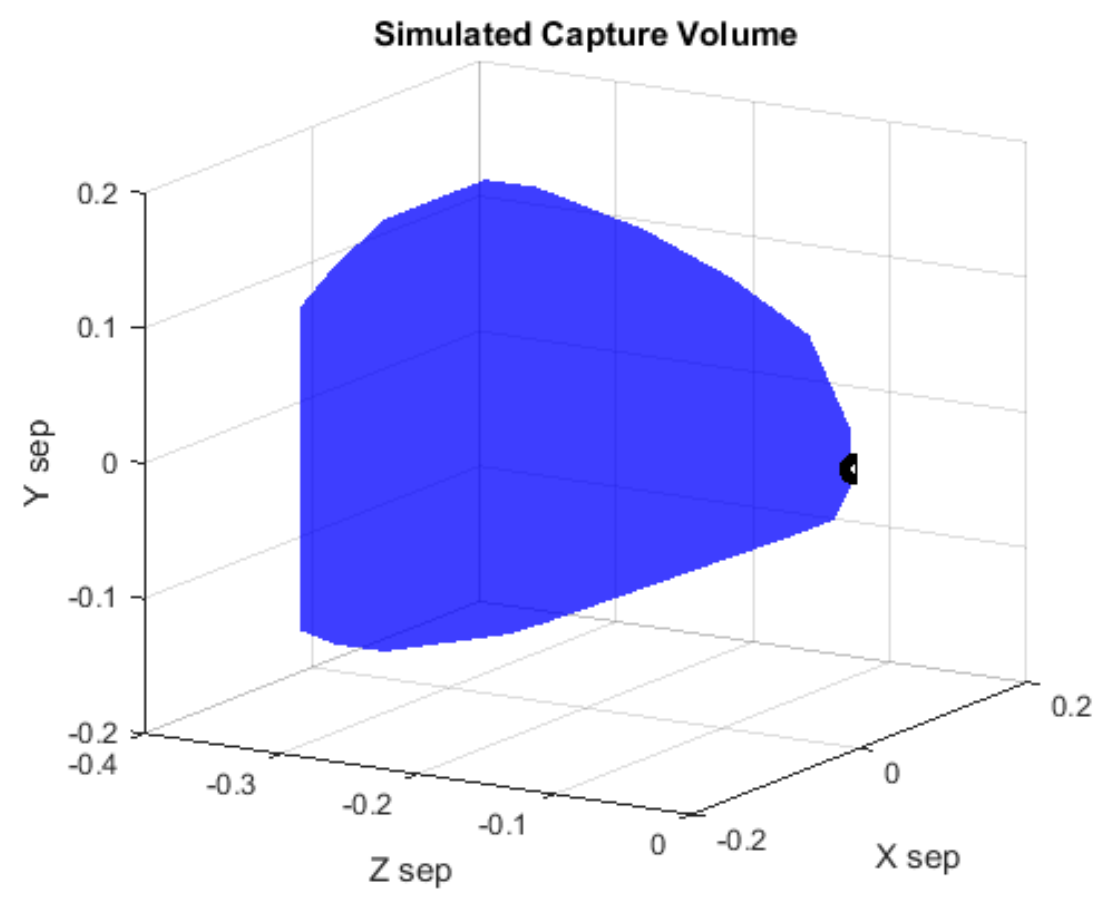

Figure 7. Simulated Capture Volume

\section{Simulation Results}

An initial 9 DOF docking simulation was created with best estimates of key system uncertainties including CDGPS relative position bias, noise, thruster MIB, attitude determination errors, etc. Clohessy-Wiltshire equations ${ }^{17}$ were used to model the relative translational dynamics in space. There have been numerous studies that explore docking controller strategies. ${ }^{18-21}$ For this project, a low bandwidth full state feedback docking controller was designed to bring the follower CubeSat towards the leader $\mathrm{O}(\mathrm{cm} / \mathrm{s})$. This is based on the assumption that the far field rendezvous GNC algorithm has successfully brought the two CubeSats within an adequately close proximity, $\mathrm{O}(\mathrm{m})$. The relative bandwidth between the radial and axial channel is approximately 4 to 1 with the rationale that any excessive radial velocity components needs to be eliminated prior to the CubeSat entering the magnetic basin of attraction. A nonlinear phase plane controller also seemed to work well by giving the designer the option to wisely choose the desired coast velocity, position and velocity dead bands etc. in each channel with the magnetic docking subsystem capture volume in mind.

The docking simulation starts when the CubeSats are within $4 \mathrm{~m}$ of each other in the v-bar direction with up to $2 \mathrm{~m}$ of radial offset. Termination conditions are: 1) Successful docking, when the centerline of the docking interfaces are within a $1 \mathrm{~cm}$ offset radius at an axial separation distance of $1 \mathrm{~cm}$ within minimal attitude rates. 2) Unsuccessful docking, when the follower overshoots the leader or when the magnetic docking subsystem generates large attitude motions in the CubeSats due to excessive misalignment errors between the docking subsystems. Figures 8 and 9 are time history plots of the attitude quaternion (first element being the scalar) and relative position (locations of the docking subsystem center in the LVLH 
frame) for a successful docking scenario. The red dashed line in Figure 9 represents the target location at which feedback control is turned off. The $X$ and $Z$ body axes are aligned with the local r-bar and v-bar directions respectively. In this run, there was a $6 \mathrm{~cm}$ and $4 \mathrm{~cm}$ constant bias in the CDGPS $X$ and $Y$ channel relative position measurements. The follower CubeSats enters the magnetic cone of attraction with small radial velocity components and attitude errors. The magnetic docking subsystem subsequently corrects for the position offset caused by the CDGPS bias, aligns the two CubeSats, and finally snaps them in place.
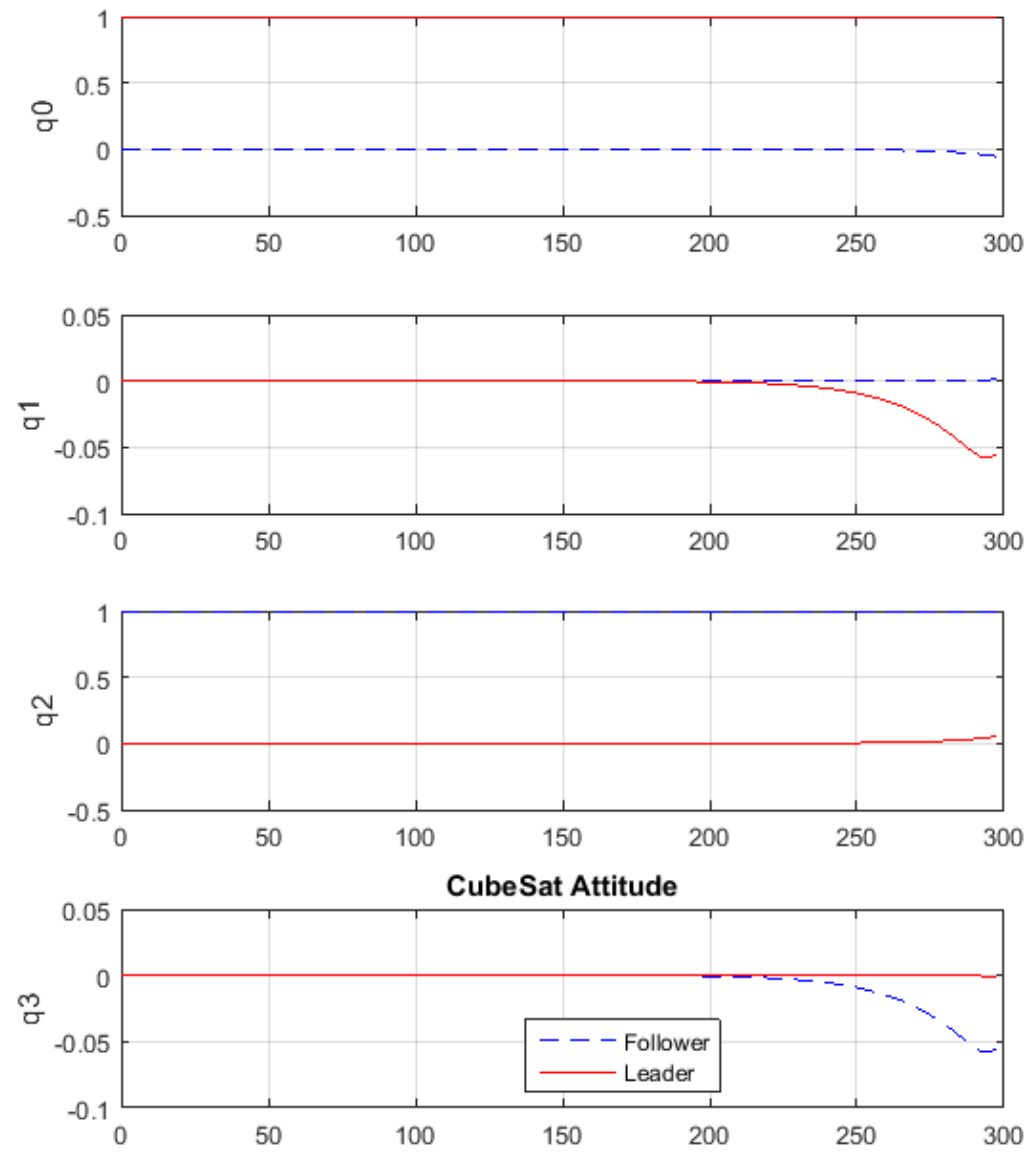

Figure 8. Successful Docking Example: Attitude Time History 

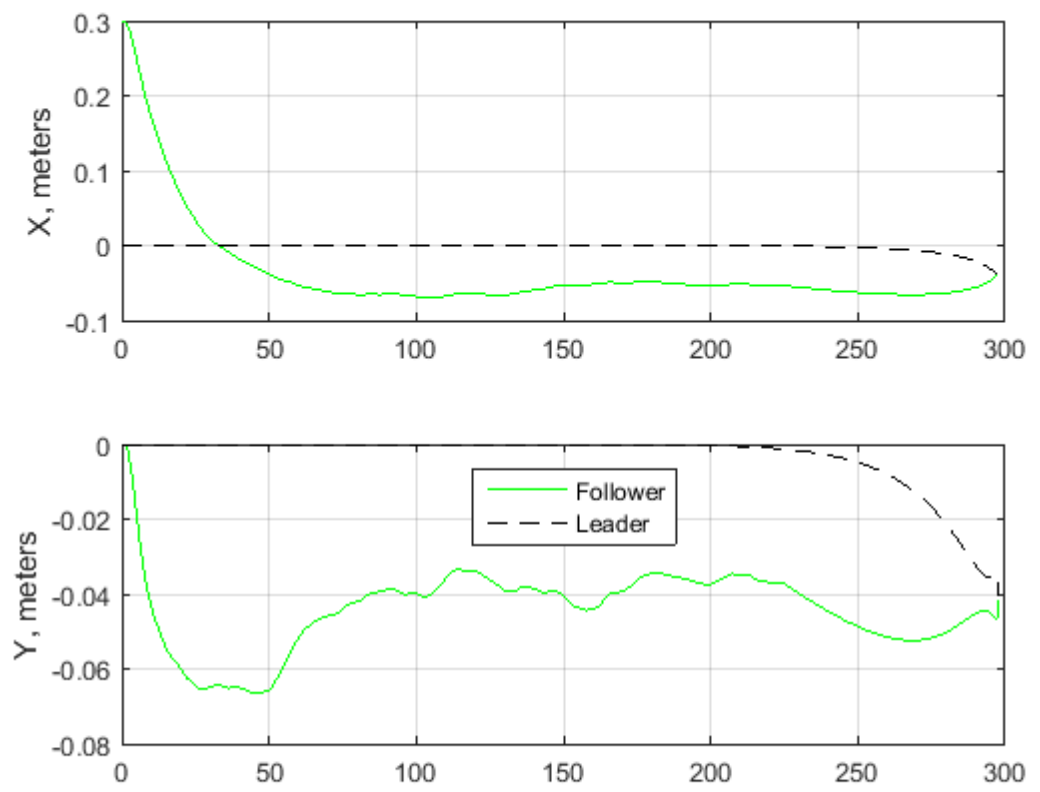

Relative Position Between Docking Interfaces

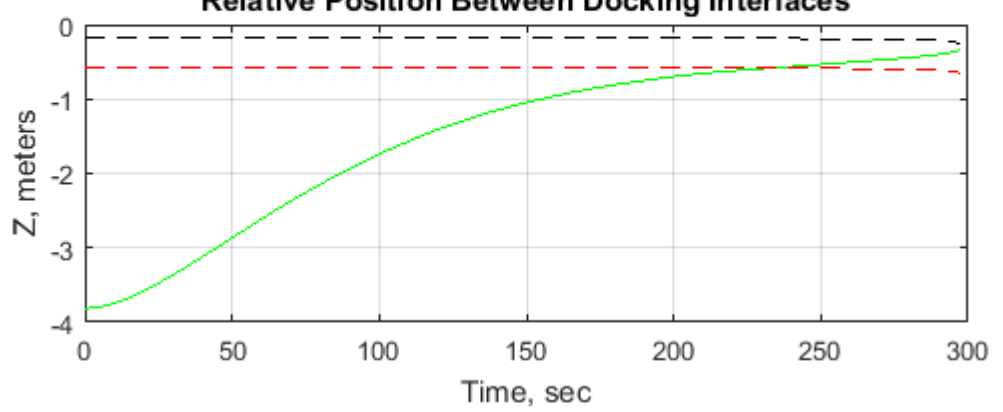

Figure 9. Successful Docking Example: Relative Position Time History

Figure 10 is a Monte Carlo plot showing the relative offset between the centerline of the two docking interfaces (red dots) at an axial separation of $1 \mathrm{~cm}$. If the dots fall within the green circle $(1 \mathrm{~cm}$ radius), then docking is considered a success. It is apparent that the proposed docking subsystem is robust to $3 \sigma$ uncertainties in the system parameters. Figure 11 is a similar Monte Carlo simulation but without the proposed docking subsystem in place. The majority of the scenarios fall outside the green circle as expected, showing that robust docking is not possible without the aid of the proposed magnetic docking subsystem given the sensors and actuators that were chosen for this project. 


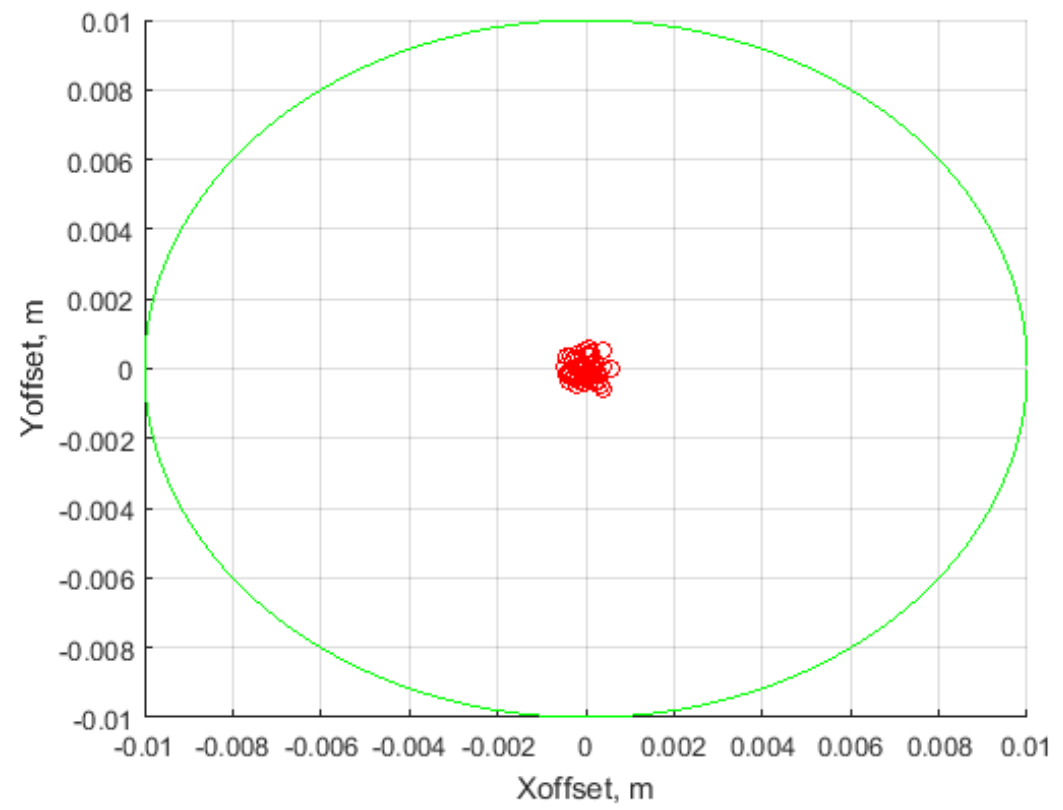

Figure 10. Monte Carlo Results: with docking subsystem

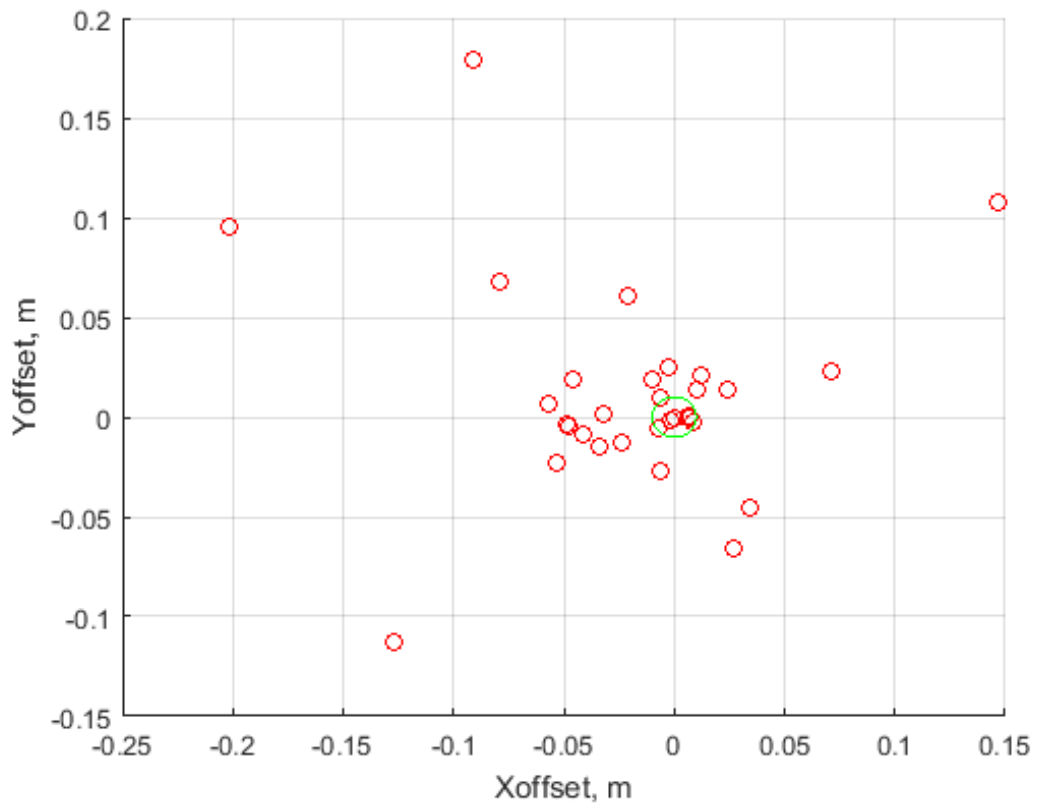

Figure 11. Monte Carlo Results: without docking subsystem

A more elegant and stricter way of determining successful docking is to compare the potential energy field created by the magnetic docking subsystems with the residual kinetic energy associated with the follower CubeSat. Equation.( 5 ) is an analytical representation of the potential energy between 2 magnetic dipoles. In the current docking design, the overall potential energy field can be represented as the sum of the potential energy between the 4 pairs of dipoles. The characteristics of the potential field becomes more complex as additional magnets are included in the design and is analogous to the concept of a $\mathrm{N}$ body orbital mechanics problem. ${ }^{22}$ Figure 12 illustrates the potential energy contours at $0.12 \mathrm{~m}, 0.14 \mathrm{~m}$, and $0.16 \mathrm{~m}$ of 
axial separation. Empty regions are where the potential energy is negative due to destructive interference between the magnetic dipoles.

$$
H=-\frac{\mu_{o}}{4 \pi|\vec{r}|^{3}}\left(3\left(m_{a} \cdot \hat{r}\right)\left(m_{b} \cdot \hat{r}\right)-\left(m_{a} \cdot m_{b}\right)\right)
$$

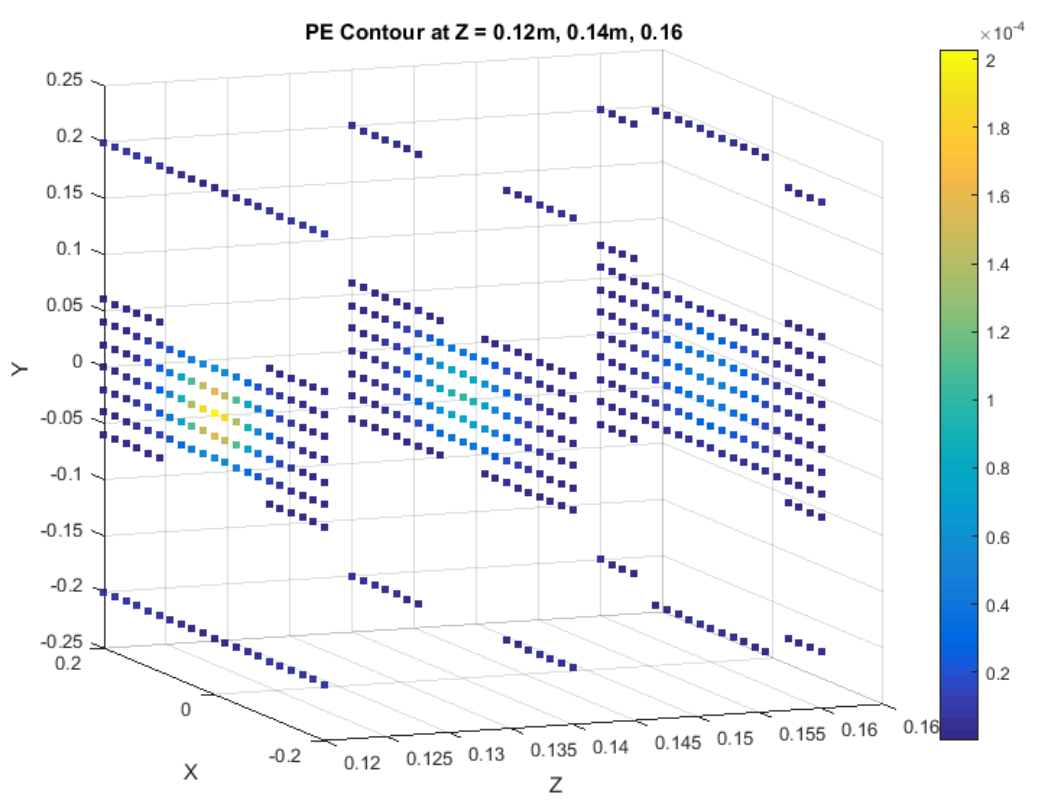

Figure 12. Docking Subsystem Potential Energy Contour at Axial Separation $=0.12 \mathrm{~m}, 0.14 \mathrm{~m}$, and $0.16 \mathrm{~m}$

In the nominal operational scenario, the GNC algorithm brings the follower CubeSat to a virtual target location directly in front of the leader CubeSat with zero kinetic energy, subsequently feedback control is turned off and the magnetic potential gradually brings the two together. Docking is guaranteed in this ideal case because the initial kinetic energy will always be smaller compare to the potential energy at the target location. However, due uncertainties in the plant dynamics such as CDGPS bias and thruster MIB the controller is not able to precisely bring the follower CubeSat to the target with the desired kinetic energy. The design objective is to pick a target location with the largest potential energy field while still ensuring robustness to CDGPS bias $(2 \mathrm{~cm}, 1 \sigma)$. For the current docking design, the target is chosen at $x=0, y=0$, $z=0.16 \mathrm{~m}$. This is the closest the target could be with respect to the leader and still be relatively robust to a $3 \sigma$ CDGPS bias. Figure 13 illustrates graphically the sphere of uncertainty (6 cm radius) associated with the CDGPS position measurement. As long as the sphere is inside the volume of positive potential energy (represented by colored dots) the system is robust to CDGPS bias in a loose sense. 


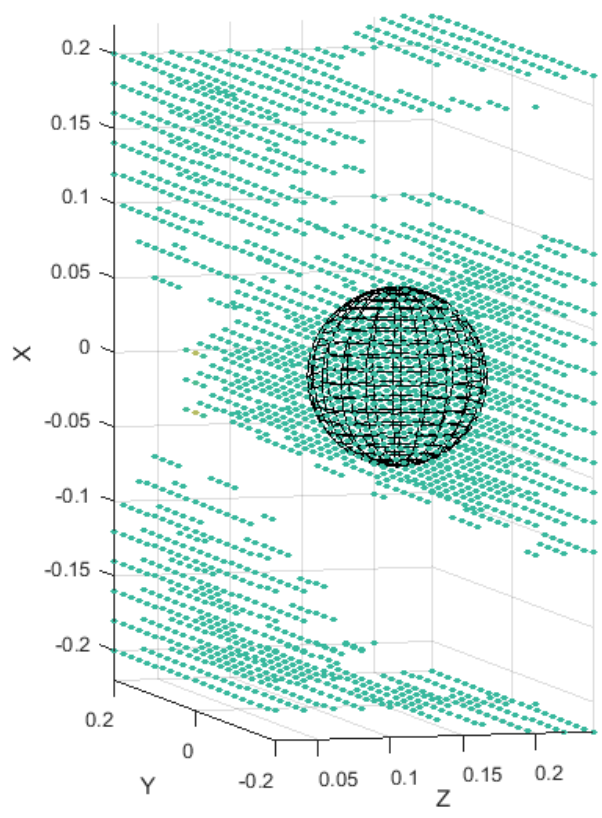

Figure 13. CDGPS Position Measurement Uncertainty

A more general way of determining successful docking in this approach is to perform Monte Carlo simulation and comparing the dispersed kinetic energy at the time feedback control is OFF with the potential energy contour of the docking subsystem. Figure 14 shows the potential energy contour of the proposed docking subsystem as a function of axial and radial separation with the blue dots representing the dispersed residual kinetic energy of the follower CubeSat. Most of the kinetic energy is below the potential energy contour which suggests that the current version of the docking design is able to guarantee successful docking from an energy point of view given the uncertainties in the plant dynamics. The few case in which the kinetic energy is above the potential energy contour can still successfully dock according the metric described by Fig. 10. The OAAN team is actively pursuing a docking design that ultimately satisfies both success metrics in a Monte Carlo sense. 


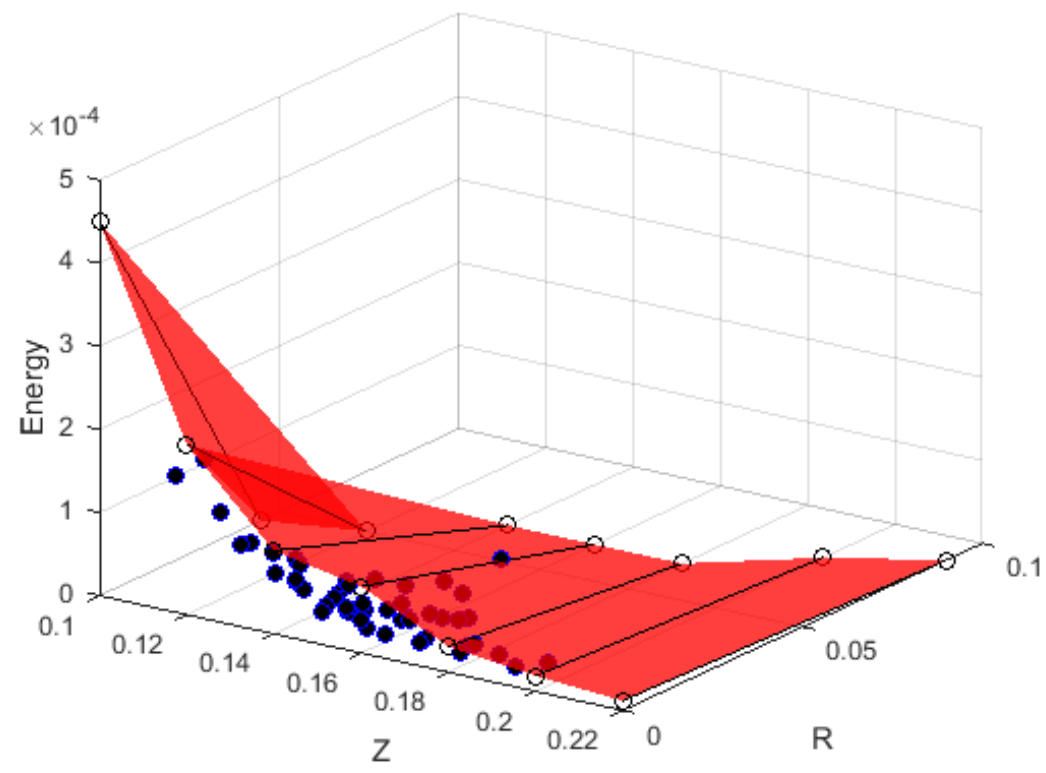

Figure 14. Dispersed Residual Kinetic Energy

\section{Conclusion}

Autonomous rendezvous and docking of two CubeSats in space is a challenging GNC problem. The OnOrbit Autonomous Assembly of Nanosatellites (OAAN) team at NASA Langley Research Center along with Cornell University have developed a novel solution to this problem using permanent magnets which allow CubeSats to align and lock during the final docking phase. Preliminary simulation demonstrated the proposed magnetic docking concept eliminates the need for high precision sensors and actuators. The mechanical team is currently improving the docking subsystem for multiple docking and undocking functionalities. Work is ongoing to obtain experimental verification of the performance of the docking hardware, cold gas thrusters, navigation sensors, and to demonstrate the flight-like system on a flat floor facility at NASA Langley Research Center.

\section{Acknowledgement}

The authors would like thank the NASA Science Technology Mission Directorate (STMD) for funding to pursue this concept. The authors would also like to extend their gratitude to Dr. Mason Peck, Dr. Carlos Roithmayr, and Dr. Bandu Pamadi for their encouragement, mentoring and insight.

\section{References}

${ }^{1}$ Zeledon, R. and Peck, M., "Attitude Dynamics and Control of a 3U CubeSat with Electrolysis Propulsion," AIAA, 2013.

${ }^{2}$ Franquiz, F. and Edwards, P., "Attitude Determination and Control System Design for a 6U CubeSat for Proximity Operations and Rendezvous," No. 2014-4421, AIAA, 2014.

${ }^{3}$ Y. Yoo, G. K. and Koo, S., "Attitude Control System of a Cube Satellite with Small Solar Sail," No. 2015-0939, AIAA SciTech, 2015.

${ }^{4}$ Lightsey, E. and Fear, A., "CubeSat Autonomous Rendezvous Simulation," No. 2015-0318, AIAA SciTech, 2015.

${ }^{5}$ Tisa, P. and Vergez, P., "Performance Analysis of Control Algorithms for FalconSat-3," No. AAS 06-149, AAS, 2006.

${ }^{6}$ J. Bowen, A. Tsuda, J. A. and Villa, M., "CubeSat Proximity Operations Demonstration Mission Update," IEEE, 2015.

${ }^{7}$ M. Nunes, T. S. and Pilger, E., "On the development of a 6DoF GNC framework for docking multiple small satellites," No. 2015-0868, AIAA SciTech, 2015.

${ }^{8}$ Underwood, J. and Pellegrino, C., "Auntonomous Assembly of a Reconfigurable Space Telescope - A CubeSat/Microsatellite Based Technology Demonstrator," No. SSC-VI-5, AIAA/USU Conference on Small Satellites, 2013.

${ }^{9}$ J. Underwood, C. Pellegrino, V. L. C. B. and Baker, J., "Using CubeSat/micro-satellite technology to demonstrate the 
Autonomous Assembly of a Reconfigurable Space Telescope," Acta Astronautica, 2015.

${ }^{10}$ Polites, M., "Technology of Automated Rendezvous and Capture in Space," Journal of Spacecraft and Rockets, Vol. 36, No. 2, March-April 1999.

${ }^{11}$ NASA.gov, "Overview of the DART Mishap Investigation Results," 2006.

${ }^{12}$ SwiftNav, Piksi DataSheet, June 2013.

${ }^{13}$ Blue Canyon Technologies, XACT DataSheet, Feb 2015.

${ }^{14} \mathrm{M}, \mathrm{S} .$, Spacecraft Dynamics and Control, Cambridge University Press, 5th ed., 2006.

${ }^{15}$ Yung. K, Landecker. P, V. D., "An Analytical Solution for the Force Between Two Magnetic Dipoles," Magnetic and Electrical Separation, Vol. 9, 1998, pp. 39-52.

${ }^{16}$ Yung. K, Landecker. P, V. D., "An Analytical Solution for the Torque Between Two Magnetic Dipoles," Magnetic and Electrical Separation, Vol. 10, 1999, pp. 29-33.

${ }^{17}$ Clohessy, W. and Wiltshire, R., "Terminal Guidance System for Satellite Rendezvous," Journal of the Aerospace Sciences, Vol. 27, No. 9, 1960, pp. 653-658.

${ }^{18}$ P. Singla, K. S. and Junkin, J., "Adaptive Output Feedback Control for Spacecraft Rendezvous and Docking Under Measurement Uncertainty," Journal of Guidance, Control, and Dynamics, Vol. 29, No. 4, July-August 2006.

${ }^{19}$ Kluever, C., "Feeback Control for Spacecraft Rendezvous and Docking," Journal of Guidance, Control, and Dynamics, Vol. 22, No. 4, July-August 1999

${ }^{20}$ C. Tournes, Y. S. and Foreman, D., Sliding Mode Control, InTech, 2011.

${ }^{21}$ S. Cairano, H. P. and Kolmanovsky, I., "Model Predictive Control Approach for Guidance of Spacecraft Rendezvous and Proximity Maneuvering," International Journal of Robust and Nonlinear Control, Vol. 22, May 2012, pp. 1398-1427.

${ }^{22}$ Vallado, D. and Wayne, M., Fundamentals of Astrodynamics and Applications, McGraw-Hill, 2001. 